\title{
STesearch Square
}

\section{Comparison of the Bone Cement Leakage on}

Vertebroplasty with High-Viscosity Cement Versus Decompressed Kyphoplasty: A Retrospective Study

\section{Shih-Hao Cheng}

National Yang Ming Chiao Tung University

\section{Wen-Hsiang Chou}

Cheng Hsin General Hospital

\section{Yu-Chuan Tsuei}

National Yang Ming Chiao Tung University

\section{Shih-Sheng Sun}

Cheng Hsin General Hospital

\section{Man-Kuan Au}

Cheng Hsin General Hospital

\section{William Chu}

National Yang Ming Chiao Tung University

Woei-Chyn Chu ( $\nabla$ wchu@ym.edu.tw)

National Yang Ming Chiao Tung University

\section{Research Article}

Keywords: cement leakage, high viscosity cement, kyphoplasty, osteoporotic compression fracture, vertebroplasty

Posted Date: October 25th, 2021

DOI: https://doi.org/10.21203/rs.3.rs-962372/v1

License: (a) (1) This work is licensed under a Creative Commons Attribution 4.0 International License. Read Full License 


\section{Abstract}

Cement leakage is a concerning complication of percutaneous kyphoplasty or vertebroplasty, as it may lead to neurologic injury. High viscosity cement has been recently used in vertebroplasty to prevent cement leakage. In this study, we aimed to compare the results between vertebroplasty with high viscosity cement to a novel technique "decompressed kyphoplasty" that used a low viscosity cement. A total of 136 patients with a single level osteoporotic compression fracture were retrospectively included in our study. 70 patients received vertebroplasty with high viscosity cement while the other 66 patients received decompressed kyphoplasty with low viscosity cement. The overall cement leakage rate was not significantly different between the two groups $(18.6 \%$ and $9.1 \%, p=0.111)$. However, the risk of intradiscal leakage was lower for the decompressed kyphoplasty group $(p=0.011)$, especially when the pre-operative $x$-ray did not display a cleft sign. The change in the kyphotic angle and the risk of adjacent segment collapse were similar ( $p=0.739$ and 0.522 respectively). We concluded that decompressed kyphoplasty can reduce intradiscal cement leakage rate compared to high viscosity cement vertebroplasty. The technique is particularly useful for patient without cleft sign on $\mathrm{x}$-ray to prevent intradiscal leakage.

\section{Introduction}

Osteoporotic compression fractures are a common occurrence in the geriatric population, leading to disability and great social burden ${ }^{1-3}$. Although most compression fractures can be managed conservatively, some cause intolerable symptoms and require surgical treatment ${ }^{4,5}$. Percutaneous vertebroplasty and kyphoplasty have been used to treat symptomatic osteoporotic compression fractures worldwide, and have demonstrated satisfactory results ${ }^{6-8}$. Although the procedure provides good clinical outcomes, the risk of cement leakage is constantly an important concern ${ }^{9,10}$. Studies have reported that the risk of cement leakage is underestimated, and was higher than we have observed on radiographic examination ${ }^{9}$. Although most leakages are asymptomatic, some can lead to devastating complications, such as neurologic injury and pulmonary embolism ${ }^{11}$. Therefore, surgeons put great effort into identifying risk factors and reducing the occurrence of cement leakage. In earlier research, factors including fracture patterns, surgical technique, injury duration, and cement viscosity, were identified to be factors affecting cement leakage ${ }^{12-14}$. Recently, vertebroplasty using high-viscosity cement was used to treat osteoporotic compression fractures. This type of cement has a shorter liquid phase, and was claimed to be more predictable during injection, thus reducing the risk of cement leakage ${ }^{15-18}$. However, the effectiveness of high-viscosity cement is not well understood and there is a lack of data regarding long-term outcomes. The distribution of the cement inside the vertebral body is of additional concern. The cement cannot penetrate into the cancellous bone and medullary canal as effectively as the cement of lower viscosity ${ }^{19,20}$. Uneven distribution and filling of the cement may lead to poorer biomechanical strength ${ }^{21-24}$, and may increase subsequent adjacent segment collapse. There are few researches reporting the kyphotic angle change and adjacent segment fractures in literature review. 
In the previous study we described the use of a novel differential pressure technique as a driving force to lead a "low viscosity" bone cement flowing through the vertebral fracture site. Our results showed that a significant decrease in bone cement leakage can be achieved ${ }^{25}$. In this study, we aimed to compare the risk of the cement leakage rate, radiographic outcome and rate of adjacent segment fracture between vertebroplasty with high-viscosity cement and decompressed kyphoplasty with low-viscosity cement.

\section{Methods}

\section{Study design}

We retrospectively reviewed the patients who received vertebroplasty or kyphoplasty in our hospital from March 1, 2017 to March 1, 2019. Pre-operative and post-operative imaging, charts and surgery reports were reviewed. All of the surgeries were performed by the same surgeon with over 20 years' experience in spinal surgery. The current study was approved by the institutional review board of Cheng Hsin general hospital (IRB No.: 778109-17). The study was performed in accordance with the relevant guidelines and regulations. An informed consent was obtained from all participants

\section{Patient inclusion criteria}

Each patient included in the study had a single level osteoporotic compression fracture and received either vertebroplasty with high-viscosity cement (Confidence ${ }^{\circledR}$, Johnson \& Johnson, New Jersey, USA) or decompressed kyphoplasty with low-viscosity cement (Simplex Bone Cement Radiopaque, Howmedica, New Jersey, USA ). All patients were diagnosed based on clinical symptoms, plain-film x-rays and magnetic resonance image (MRI) prior to surgery. There must have been at least four weeks of conservative treatment with medicine or bracing before surgery. All patients were followed up more than 12 months after the surgery.

\section{Exclusion criteria}

Patients who suffered from high energy traumatic compression fracture, pathologic compression fractures caused by tumor, old compression fractures (more than three months) with union, and burst fractures were excluded. Patients who had multiple-level procedures were precluded from analysis as well. If there were any previous compression fractures or spinal instrumentation over adjacent level, the patient was also excluded as these might affect the risk of adjacent compression fracture. Patients who did not have radiographic follow-up for more than 12 months were also excluded.

\section{Surgical technique}

\section{Decompressed kyphoplasty}

We used the decompressed technique as reported in a previous article to guide the direction of the cement flow ${ }^{25}$. All procedures were performed under general anesthesia or intravenous sedation anesthesia. The patient was placed in the prone position on a radiolucent table, and two 8 gauge needles 
were introduced through bilateral pedicles into the vertebral body. The kyphoplasty balloon (Medtronic, Minnesota, USA) was inserted into the collapsed body and inflated from each trocar in order to reduce the collapsed endplate. After the pressure reach $200 \mathrm{Psi}$, or once the fracture was reduced, the balloon was deflated and removed. Low-viscosity cement was prepared at room temperature, and the viscosity of the cement was confirmed using the spaghetti method. Built-in suction in the operating room was connected to one trocar in order to create negative pressure, and the bone cement was installed from the other side manually. When the cement fully occupied the fracture site, or cement no longer emerged from the decompressed trocar anymore, the installation of cement was stopped, and the trocars were removed. The whole procedure was performed under fluoroscopic guidance. The details of the technique were provided in our previous work ${ }^{25}$.

\section{Vertebroplasty with high viscosity cement}

The positioning of the patient, anesthesia methods and surgical approach were the same for both groups, except that the 13 gauge trocars were inserted through bilateral pedicles instead. High-viscosity cement was then injected from trocars using a hydraulic delivery system that came with the package. The endpoint of cement injection was achieved with complete cement filling of the fracture site.

\section{Radiographic results}

Pre-operative and post-operative kyphotic angle and cement leakage were evaluated on x-ray. The kyphotic angle was defined as the angle between the extension of the upper and lower endplates, which was measured independently by two authors; an average value was then calculated. Presence of cement leakage, leakage type and occurrence of an adjacent segment compression fracture were also determined by the same two authors. Cement leakage was further divided into four groups according to location, including paravertebral, intradiscal, epidural and intra-venous types. The cleft sign, or vacuum phenomenon (Figure 1), was defined as a radiolucent linear or semilunar shadow on either the frontal or lateral $x$-ray ${ }^{26}$. Any discrepancies related to $x$-ray interpretation were discussed by the two authors, who reached a consensus. If an agreement could not be reached, a third author was consulted and made the final decision.

\section{Statistics}

The data was processed with SPSS 22 (IBM Corporation, New York, USA). The general patient information was presented with descriptive statistics. Category variables, such as gender, presentation of cement leakage and adjacent segment collapse were compared using the Chi-square test. We compared the continued variables, including age, kyphotic angle changes and follow-up duration between the two groups with an independent sample t-test. The kyphotic angle difference before and after operation was compared with a dependent sample t-test. Subgroup analysis was performed in the group receiving the high viscosity cement, in order to determine the effect of the vacuum phenomenon. Testing for normality was performed using the built-in function of SPSS software. The abovementioned data were assessed 
visually with a normal Q-Q plot and numerically with a Shapiro-Wilk test of normality, which showed normally distributed data. A p-value less than 0.05 was considered statistically significant.

\section{Results}

\section{Patient demographics}

A total of 136 patients, 23 males and 113 females, were included in our study. 70 patients received vertebroplasty with high-viscosity cement (Group 1) and 66 patients received decompressed kyphoplasty with low-viscosity cement (Group 2). There was no significant difference in age, sex, and follow-up duration between two groups. Most of the operated levels are between T11 to L2 (Table 1).

\section{Radiography results}

Both groups showed significant improvement in the kyphotic angle after the surgery $(p<0.01)$. On the contrary, the kyphotic angle before and after surgery were similar between the two groups $(p=0.551, p=$ 0.651 , respectively). The kyphotic angle changes were not significant $(p=0.739)$. There were 13 patients in group 1 and 6 patients in group 2 that demonstrated radiographic leakage. The overall leakage rate did not reach a significant level for the two groups $(p=0.111)$. However, there were nine patients in group 1 that showed intradiscal leakage (Figure 2), which is significantly higher than the single patient in group $2(P=0.011)$. Of note, all intradiscal leakage occurred in patients whose preoperative x-ray did not show a cleft sign, which is significant in subgroup analysis $(p=0.002)$. There was no epidural leakage or intravenous leakage in either group (Table 2).

\section{Complications}

There were no serious complications, e.g. symptomatic pulmonary embolism, neurologic symptoms or infection in either group. During follow-up, 18 adjacent segment fractures (13.2\%) occurred, mostly at the immediately superior level. However, there was no difference between the two groups, despite the higher intradiscal leakage rate in group 1.

\section{Discussion}

Our study reported that using decompressed kyphoplasty resulted in a lower rate of intradiscal cement leakage, but did not lead to a lower adjacent segment fracture rate. Intradiscal cement leakage is highly associated with the absence of a radiographic cleft sign when using high viscosity cement.

Both vertebroplasty or kyphoplasty were used to treat osteoporotic compression fractures for decades, with good results ${ }^{27}$. During performance of the procedure, cement leakage is a common complication, and can cause devastating consequences. According to previous studies, the leakage rate is heavily underestimated, and may be higher than $70 \%{ }^{9}$. Preventing cement leakage has always been an important issue. Recently, high-viscosity cement was applied to treat compression fractures, in an attempt to reduce 
the cement leakage rate ${ }^{16,28}$. In this present work, the leakage rate using high viscosity cement is $18.6 \%$, which is lower than previous reports regarding traditional vertebroplasty. However, we observed a significantly higher intradiscal leakage rate in the high viscosity cement group as compared to the decompressed kyphoplasty technique ${ }^{25}$. This phenomenon can be explained by the different cement driving forces of the two techniques. Cement in decompressed kyphoplasty follows the guide of the pressure gradient created by a suction force ${ }^{25}$. Once the balloon is inflated and creates a cavity in the vertebral body, it forms a corridor for the cement to reside in. After applying negative pressure from one trocar, cement installed from another trocar was drawn to the contralateral side passively under the guidance of the negative pressure. Conversely, the distribution of the high-viscosity cement is mainly dependent on active pressure provided manually by the surgeon. The cement often diffused profoundly from the tip of the trocar, and aggregated at the site with lowest resistance ${ }^{29}$. When the endplate defect existed, the cement can easily flow into the relative empty disc space ${ }^{30}$. This phenomenon might be more evident with the high-viscosity cement, which has a poorer ability to penetrate into cancellous bone ${ }^{31}$, causing a higher risk of intradiscal leakage. We also discovered an interesting finding in the subgroup analysis, in that the intradiscal leakage is highly correlated to whether the "cleft sign" is observed on $\mathrm{x}$-ray. The risk of intradiscal leakage is higher when the pre-operative radiography does not have a "cleft sign" visible $(p=0.02)$. This "cleft sign" or "vacuum phenomenon" represented a pre-existing space inside the vertebral body, either occupied by gas or fluid, indicating a non-healing vertebral collapse or pseudoarthrosis ${ }^{32}$. This space could be enlarged when the patient was put into a prone position and postural reduction was performed, forming an ideal space for cement to fill ${ }^{33}$. The risk of intradiscal leakage is lower when this space presented. We speculate that it is because the cement can accumulate in the space inside vertebral body, instead of infiltrated randomly out of fracture fissures. In our study, all nine patients with intradiscal leakage occurred on the fractures which did not demonstrate a pre-existing vacuum phenomenon, with a concomitant upper endplate fracture. This suggests that patients without the vacuum phenomenon on their pre-operative x-ray are under a higher risk of intradiscal disk leakage. Historically, the relationship between the cleft sign and cement leakage in vertebroplasty had been widely discussed. Some studies indicated that a cleft sign did not affect the risk of cement leakage, but rather influencing the leakage pattern ${ }^{34,35}$. Other studies revealed a significantly lower cement leakage rate in conjunction with the presence of a cleft sign ${ }^{36,37}$. There were also papers which demonstrated the opposite results, where the cleft sign may be a risk factor for increased cement leakage ${ }^{38}$. Currently, the meta-analysis of Zhan et al reviewed the evidence regarding cleft sign and concluded that the phenomenon is a risk factor for cement leakage ${ }^{39}$. This result is contrary to our findings for high viscosity cement vertebroplasty. Since the technique for vertebroplasty is similar, we presumed that unique physical properties of high-viscosity cement may lead to a different influence of a cleft sign with vertebroplasty.

Currently, the long-term effect of intradiscal cement leakage is still not well known. This type of cement leakage does not lead to neurologic injury, but it raises the concern of adjacent segment fracture because of uneven pressure distribution ${ }^{40}$. While some studies reported that the leakage did not induce adjacent 
vertebral body collapse $\mathrm{e}^{41,42}$, others obtained opposite results ${ }^{43}$. In the meantime, many studies revealed that bone cement may be toxic to the intervertebral disc and that it accelerates degeneration ${ }^{44-46}$. In our study, we did not observe a higher rate of adjacent segment fracture for the high-viscosity cement group, despite its higher intradiscal leakage rate. However, considering bone cement's potential damage to disc tissue, we should put all effort into preventing this complication, including the selection of an appropriate technique for patients without a cleft sign on pre-operative x-rays.

The study was limited by its retrospective nature, and a well-designed prospective randomized controlled trial is necessary in order to confirm our conclusions. The patients were evaluated with plain-film radiography following their surgery, which may under-estimated the leakage rate. For adjacent segment fracture, the patients only underwent a 12 month follow-up. Although previous studies reported that adjacent segment fracture following vertebroplasty or kyphoplasty often happened within three months of the procedure ${ }^{47-49}$, a longer follow-up period may be required in order to confirm the long-term effect of the different bone cements.

\section{Conclusions}

Decompressed kyphoplasty using low-viscosity cement is associated with lower intradiscal cement leakage as compared with vertebroplasty with high-viscosity cement. Absence of the cleft sign on X-ray is a strong predictor of intradiscal cement leakage. The overall leakage difference between the two groups did not reach a significant level.

\section{Declarations}

\section{Author contribution}

SHC and WC designed the study. WHC, YCT, and SSS collected and analyzed data. SHC and WC drafted the manuscript. WC and WCC revised the manuscript. WCC supervised the whole study. All authors reviewed the manuscript.

\section{Competing interests}

The author(s) declare no competing interests.

\section{Data availability}

All data are available from corresponding author on qualified request.

\section{References}

1. Silverman, S. L. The clinical consequences of vertebral compression fracture. Bone 13 Suppl 2, S2731 (1992). 
2. Kim, D. H. \& Vaccaro, A. R. Osteoporotic compression fractures of the spine; current options and considerations for treatment. Spine J 6, 479-487 (2006).

3. Cooper, C., O'Neill, T. \& Silman, A. The epidemiology of vertebral fractures. European Vertebral Osteoporosis Study Group. Bone 14 Suppl 1, S89-97 (1993).

4. Tezer, M., Erturer, R. E., Ozturk, C., Ozturk, I. \& Kuzgun, U. Conservative treatment of fractures of the thoracolumbar spine. Int Orthop 29, 78-82 (2005).

5. Lee, H. M., Park, S. Y., Lee, S. H., Suh, S. W. \& Hong, J. Y. Comparative analysis of clinical outcomes in patients with osteoporotic vertebral compression fractures (OVCFs): conservative treatment versus balloon kyphoplasty. Spine J 12, 998-1005 (2012).

6. Barr, J. D., Barr, M. S., Lemley, T. J. \& McCann, R. M. Percutaneous vertebroplasty for pain relief and spinal stabilization. Spine (Phila Pa 1976) 25, 923-928 (2000).

7. Martin, J. B. et al. Vertebroplasty: clinical experience and follow-up results. Bone 25, 11S-15S (1999).

8. Hulme, P. A., Krebs, J., Ferguson, S. J. \& Berlemann, U. Vertebroplasty and kyphoplasty: a systematic review of 69 clinical studies. Spine (Phila Pa 1976) 31, 1983-2001 (2006).

9. Schmidt, R. et al. Cement leakage during vertebroplasty: an underestimated problem? Eur Spine J 14, 466-473 (2005).

10. Ding, J. et al. Risk factors for predicting cement leakage following percutaneous vertebroplasty for osteoporotic vertebral compression fractures. Eur Spine J 25, 3411-3417 (2016).

11. Nussbaum, D. A., Gailloud, P. \& Murphy, K. A review of complications associated with vertebroplasty and kyphoplasty as reported to the Food and Drug Administration medical device related web site. $J$ Vasc Interv Radiol 15, 1185-1192 (2004).

12. Nakano, M., Hirano, N., Ishihara, H., Kawaguchi, Y. \& Matsuura, K. Calcium phosphate cement leakage after percutaneous vertebroplasty for osteoporotic vertebral fractures: risk factor analysis for cement leakage. J Neurosurg Spine 2, 27-33 (2005).

13. Nieuwenhuijse, M. J., Van Erkel, A. R. \& Dijkstra, P. D. Cement leakage in percutaneous vertebroplasty for osteoporotic vertebral compression fractures: identification of risk factors. Spine J 11, 839-848 (2011).

14. Yeom, J. S. et al. Leakage of cement in percutaneous transpedicular vertebroplasty for painful osteoporotic compression fractures. J Bone Joint Surg Br 85, 83-89 (2003).

15. Baroud, G., Crookshank, M. \& Bohner, M. High-viscosity cement significantly enhances uniformity of cement filling in vertebroplasty: an experimental model and study on cement leakage. Spine (Phila Pa 1976) 31, 2562-2568 (2006).

16. Zhang, L. et al. A comparison of high viscosity bone cement and low viscosity bone cement vertebroplasty for severe osteoporotic vertebral compression fractures. Clin Neurol Neurosurg 129, 10-16 (2015).

17. Wang, C. H., Ma, J. Z., Zhang, C. C. \& Nie, L. Comparison of high-viscosity cement vertebroplasty and balloon kyphoplasty for the treatment of osteoporotic vertebral compression fractures. Pain 
Physician 18, E187-194 (2015).

18. Li, K. et al. Role of percutaneous vertebroplasty with high-viscosity cement in the treatment of severe osteoporotic vertebral compression fractures. Sci Rep 11, 4602 (2021).

19. Reading, A. D., McCaskie, A. W., Barnes, M. R. \& Gregg, P. J. A comparison of 2 modern femoral cementing techniques: analysis by cement-bone interface pressure measurements, computerized image analysis, and static mechanical testing. J Arthroplasty 15, 479-487 (2000).

20. Rey, R. M., Jr. et al. A study of intrusion characteristics of low viscosity cement Simplex-P and Palacos cements in a bovine cancellous bone model. Clin Orthop Relat Res, 272-278 (1987).

21. Tschirhart, C. E., Roth, S. E. \& Whyne, C. M. Biomechanical assessment of stability in the metastatic spine following percutaneous vertebroplasty: effects of cement distribution patterns and volume. $J$ Biomech 38, 1582-1590 (2005).

22. Liang, D. et al. Biomechanical effects of cement distribution in the fractured area on osteoporotic vertebral compression fractures: a three-dimensional finite element analysis. J Surg Res 195, 246256 (2015).

23. Chen, B., Li, Y., Xie, D., Yang, X. \& Zheng, Z. Comparison of unipedicular and bipedicular kyphoplasty on the stiffness and biomechanical balance of compression fractured vertebrae. Eur Spine J 20, 1272-1280 (2011).

24. Li, Y. D., Tsai, T. T., Niu, C. C. \& Lai, P. L. Cement bridging phenomenon in percutaneous vertebroplasty for adjacent vertebral compression fracture. Sci Rep 11, 10184 (2021).

25. Chu, W. et al. Decompressed percutaneous vertebroplasty: a secured bone cement delivery procedure for vertebral augmentation in osteoporotic compression fractures. Injury 44, 813-818 (2013).

26. Theodorou, D. J. The intravertebral vacuum cleft sign. Radiology 221, 787-788 (2001).

27. Lavelle, W., Carl, A., Lavelle, E. D. \& Khaleel, M. A. Vertebroplasty and kyphoplasty. Anesthesiol Clin 25, 913-928 (2007).

28. Georgy, B. A. Clinical experience with high-viscosity cements for percutaneous vertebral body augmentation: occurrence, degree, and location of cement leakage compared with kyphoplasty. AJNR Am J Neuroradiol 31, 504-508 (2010).

29. Bohner, M., Gasser, B., Baroud, G. \& Heini, P. Theoretical and experimental model to describe the injection of a polymethylmethacrylate cement into a porous structure. Biomaterials 24, 2721-2730 (2003).

30. Hong, S. J., Lee, S., Yoon, J. S., Kim, J. H. \& Park, Y. K. Analysis of intradiscal cement leakage during percutaneous vertebroplasty: multivariate study of risk factors emphasizing preoperative MR findings. J Neuroradiol 41, 195-201 (2014).

31. Bean, D. J., Hollis, J. M., Woo, S. L. \& Convery, F. R. Sustained pressurization of polymethylmethacrylate: a comparison of low- and moderate-viscosity bone cements. J Orthop Res 6, 580-584 (1988). 
32. Libicher, M. et al. The intravertebral vacuum phenomen as specific sign of osteonecrosis in vertebral compression fractures: results from a radiological and histological study. Eur Radio/ 17, 2248-2252 (2007).

33. Chin, D. K., Kim, Y. S., Cho, Y. E. \& Shin, J. J. Efficacy of postural reduction in osteoporotic vertebral compression fractures followed by percutaneous vertebroplasty. Neurosurgery 58, 695-700; discussion 695-700 (2006).

34. Tanigawa, N. et al. Cement leakage in percutaneous vertebroplasty for osteoporotic compression fractures with or without intravertebral clefts. AJR Am J Roentgenol 193, W442-445 (2009).

35. Jung, J. Y., Lee, M. H. \& Ahn, J. M. Leakage of polymethylmethacrylate in percutaneous vertebroplasty: comparison of osteoporotic vertebral compression fractures with and without an intravertebral vacuum cleft. J Comput Assist Tomogr 30, 501-506 (2006).

36. Krauss, M., Hirschfelder, H., Tomandl, B., Lichti, G. \& Bar, I. Kyphosis reduction and the rate of cement leaks after vertebroplasty of intravertebral clefts. Eur Radio/ 16, 1015-1021 (2006).

37. Hiwatashi, A., Ohgiya, Y., Kakimoto, N. \& Westesson, P. L. Cement leakage during vertebroplasty can be predicted on preoperative MRI. AJR Am J Roentgenol 188, 1089-1093 (2007).

38. Ha, K. Y., Lee, J. S., Kim, K. W. \& Chon, J. S. Percutaneous vertebroplasty for vertebral compression fractures with and without intravertebral clefts. J Bone Joint Surg Br 88, 629-633 (2006).

39. Zhan, Y., Jiang, J., Liao, H., Tan, H. \& Yang, K. Risk Factors for Cement Leakage After Vertebroplasty or Kyphoplasty: A Meta-Analysis of Published Evidence. World Neurosurg 101, 633-642 (2017).

40. Kim, M. H., Lee, A. S., Min, S. H. \& Yoon, S. H. Risk factors of new compression fractures in adjacent vertebrae after percutaneous vertebroplasty. Asian Spine J 5, 180-187 (2011).

41. Lee, K. A. et al. Analysis of adjacent fracture after percutaneous vertebroplasty: does intradiscal cement leakage really increase the risk of adjacent vertebral fracture? Skeletal Radiol 40, 1537-1542 (2011).

42. Pitton, M. B. et al. CT-guided vertebroplasty in osteoprotic vertebral fractures: incidence of secondary fractures and impact of intradiscal cement leakages during follow-up. Eur Radiol 18, 43-50 (2008).

43. Lin, E. P., Ekholm, S., Hiwatashi, A. \& Westesson, P. L. Vertebroplasty: cement leakage into the disc increases the risk of new fracture of adjacent vertebral body. AJNR Am J Neuroradio/ 25, 175-180 (2004).

44. Jamjoom, B., Patel, S., Bommireddy, R. \& Klezl, Z. Impact of the quantity of intradiscal cement leak on the progression of intervertebral disc degeneration. Ann R Coll Surg Eng/ 99, 529-533 (2017).

45. Shen, M. et al. Adjacent disc height reduction and clinical outcome after intradiscal cement leakage. Int J Spine Surg 10, 34 (2016).

46. Mao, H. et al. Intervertebral disc degeneration induced by intradiscal poly(methyl methacrylate) leakage after spine augmentation in an in vivo rabbit model. Acta Biomater 10, 3059-3067 (2014).

47. Takahara, K. et al. Risk factors of adjacent vertebral collapse after percutaneous vertebroplasty for osteoporotic vertebral fracture in postmenopausal women. BMC Musculoskelet Disord 17, 12 (2016). 
48. Rho, Y. J., Choe, W. J. \& Chun, Y. I. Risk factors predicting the new symptomatic vertebral compression fractures after percutaneous vertebroplasty or kyphoplasty. Eur Spine J 21, 905-911 (2012).

49. Trout, A. T., Kallmes, D. F. \& Kaufmann, T. J. New fractures after vertebroplasty: adjacent fractures occur significantly sooner. AJNR Am J Neuroradiol 27, 217-223 (2006).

\section{Tables}

\section{Table 1 Demographic of patients}

\begin{tabular}{|c|c|c|c|c|}
\hline & Group $1(n=70)$ & Group $2(n=66)$ & \multirow[t]{2}{*}{$p$ value } \\
\hline & & High-viscosity cement & Decompressed kyphoplasty & \\
\hline \multicolumn{2}{|l|}{ Age } & $76.46 \pm 8.38$ & $78.52 \pm 7.18$ & 0.120 \\
\hline \multicolumn{2}{|c|}{ Sex (male/female) } & $8 / 62$ & $15 / 51$ & 0.109 \\
\hline \multicolumn{2}{|c|}{ Follow up (days) } & $524.7 \pm 96.5$ & $531.9 \pm 94.6$ & 0.656 \\
\hline \multirow[t]{6}{*}{ Injured level } & Above T11 & $2(2.8 \%)$ & $3(4.5 \%)$ & \multirow[t]{6}{*}{0.854} \\
\hline & T11 & $16(22.9 \%)$ & $14(21.2 \%)$ & \\
\hline & $\mathrm{T} 12$ & $24(34.3 \%)$ & $22(33.3 \%)$ & \\
\hline & L1 & $17(24.3 \%)$ & $12(18.2 \%)$ & \\
\hline & L2 & $8(11.4 \%)$ & $12(18.2 \%)$ & \\
\hline & Below L2 & $3(4.3 \%)$ & $3(4.5 \%)$ & \\
\hline \multicolumn{2}{|c|}{ Operation time (minutes) } & $29.82 \pm 7.71$ & $40.21 \pm 12.51$ & $<0.001$ \\
\hline
\end{tabular}

\section{Table 2 Radiographic results}




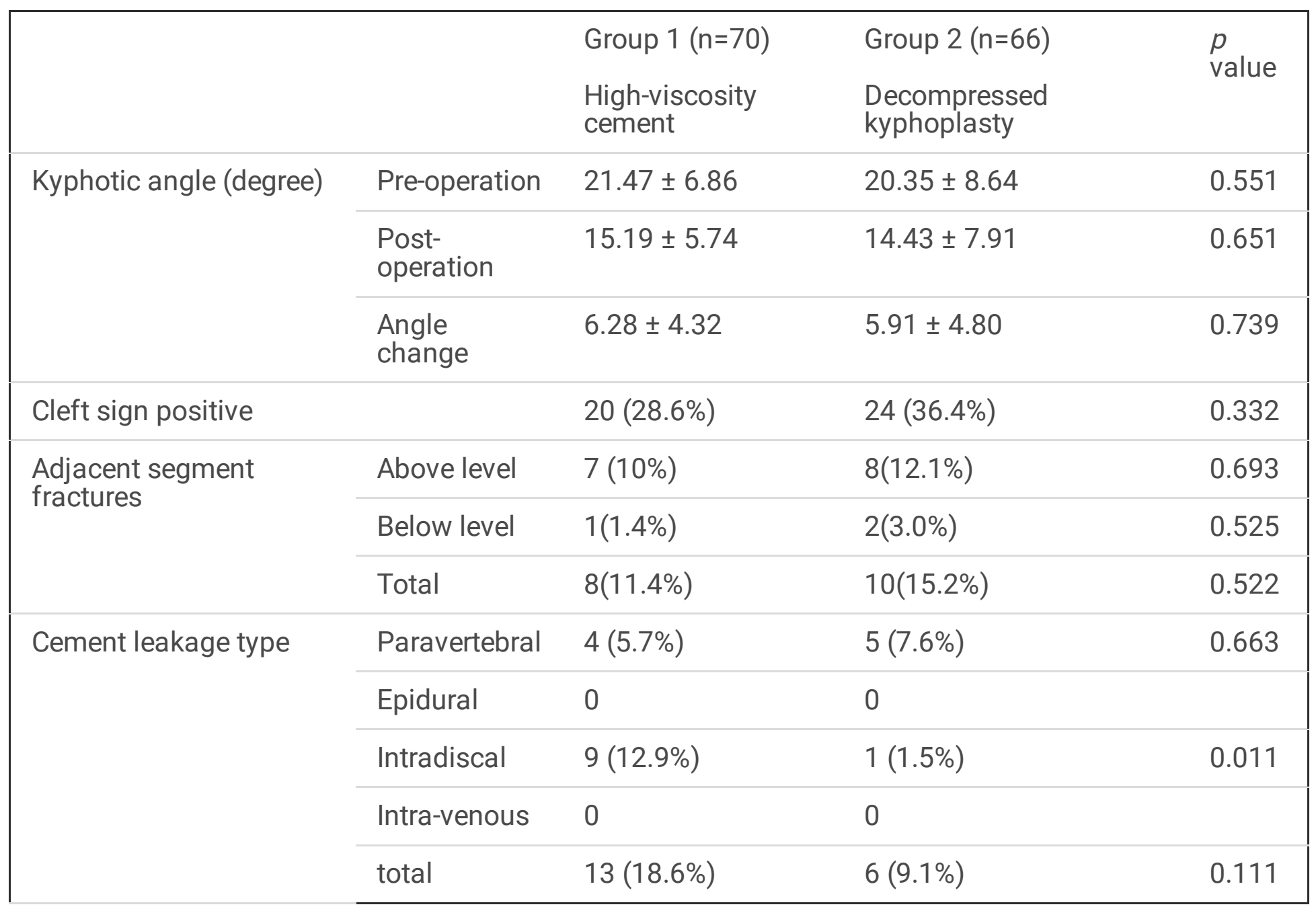

Figures 


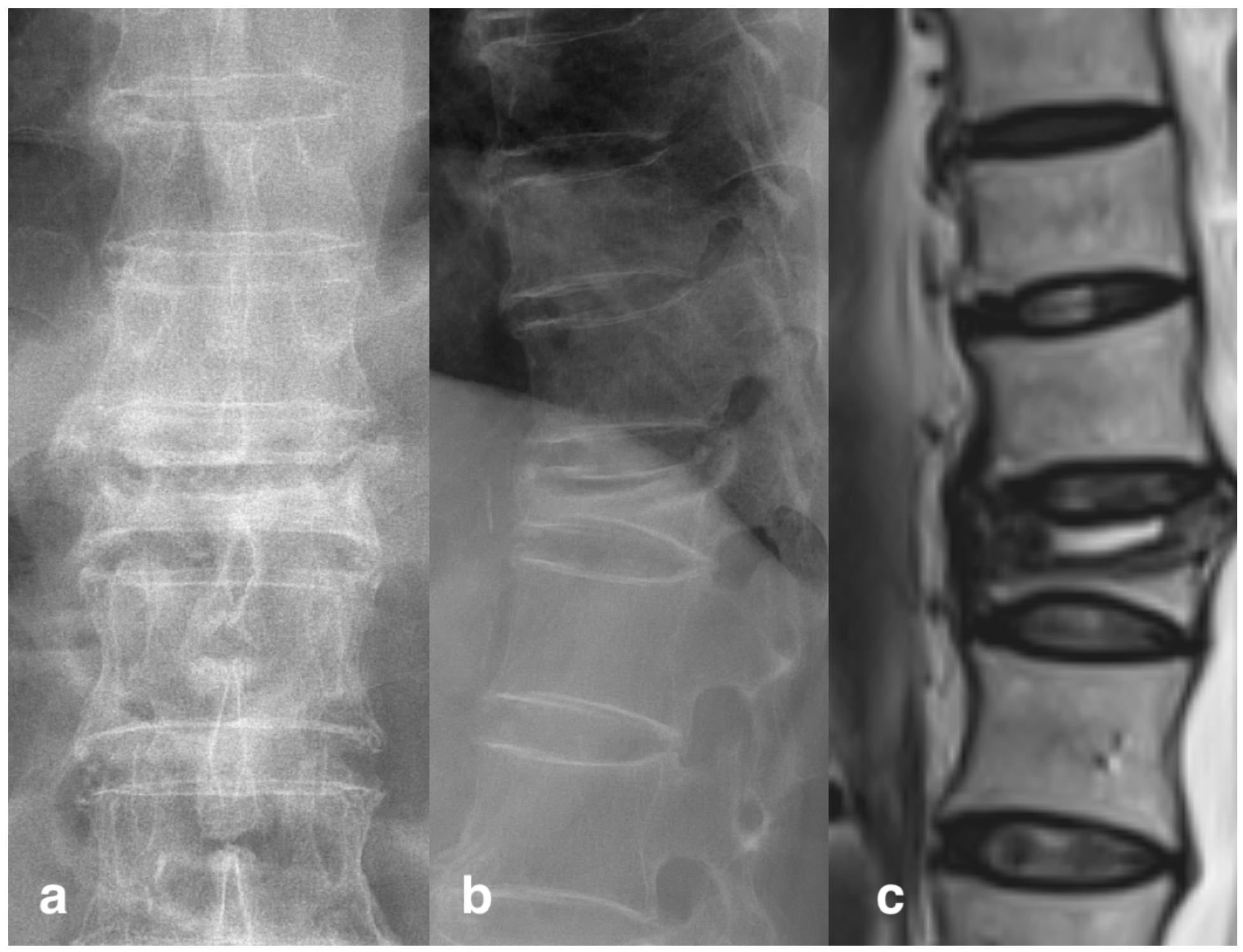

\section{Figure 1}

vertebral cleft sign The radiography of a patient with $\mathrm{T} 12$ compression fracture showed a typical cleft sign on (a) AP view and (b) lateral view. The sagittal view on T2 weight imaging MRI (c) also revealed a fluid-contained empty space in vertebral body. 


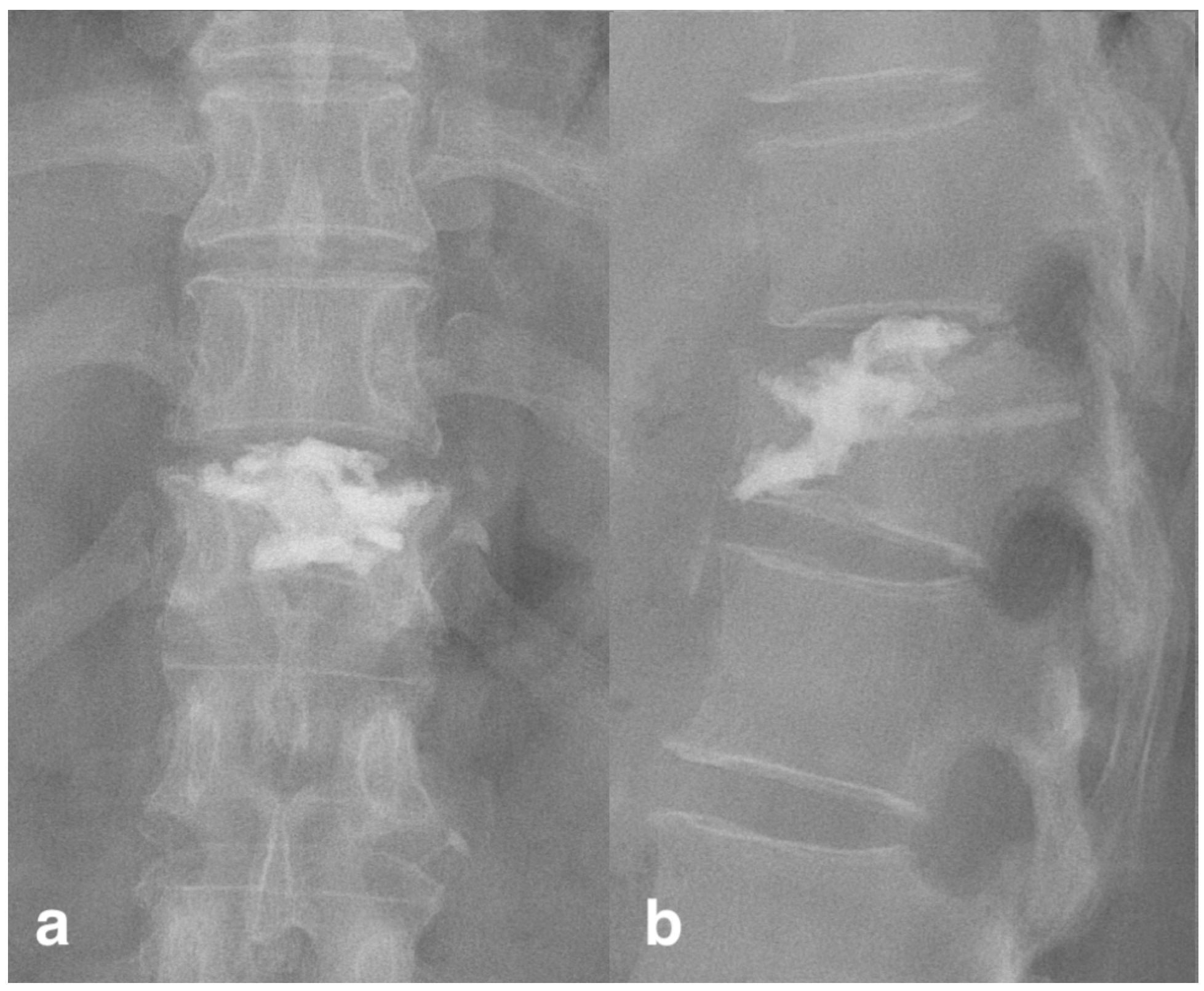

\section{Figure 2}

Intradiscal cement leakage A 61 year-old female received vertebroplasty with high viscosity cement because of the compression fracture of T12. Postoperative $X$ ray showed intradiscal cement leakage on both (a) AP view and (b) lateral view. 